\title{
Research and implementation of network fault reduction based on Virtual platform
}

\author{
Zhenhua Chai 1 ,a \\ ${ }^{1}$ State Grid Jibei Electric power company Chengde power supply company Chengde067000 China \\ a1248807382@qq.com
}

Keyword: virtualplatform,Network topology, Equipment downtime,Fault reduction

\begin{abstract}
With the continuous development of the state grid, the application of information technology has been strengthened. And the application of data network is more and more complex. In this paper, it will combine the practical work experience with the fault location and the exclusion of the state grid data network. The design and feasibility verification of project details are discussed in this paper. Proposed the establishment of a comprehensive data network virtual test platform to simulate the real network environment, in the simulation environment to achieve the rapid positioning of the fault, verification solutions, rapid implementation, improve the ability to deal with the fault, shorten the time of fault investigation, detailed implementation steps, so that the network system has always been maintained, can control, in control.
\end{abstract}

\section{Introduction}

The company's comprehensive data network was founded in 2006, is the important part of the state grid network platform. At present, it has covered all $35 \mathrm{kV}$ and above substations. Responsible for the company's production, business information application systems, video conferencing systems, video surveillance systems and other business data transmission, the entire network such as the company's nerve center, plays a pivotal role.

Throughnearly 10 years of expansion, the company has developed into a huge system network . Although it played a strong supporting role in the operation of the company, but in the actual application of integrated data network, there are many network problems such as the slow convergence, the device compatibility between network equipment and so on. At the same time, in the actual fault investigation or project implementation, it will cause network oscillations, the network is not smooth and even the risk of collapse

\section{Research purposes}

Network is the most basic guarantee of the operation of the business system. That the network been not smooth, will lead to a comprehensive application system paralysis. Therefore, it is very important to find out a reasonable and scientific method to deal with the fault of the data network and simulate the real application.

Chengde power supply company based on its own network, and strive to explore governance means, through continuous exploration, through the virtual network platform, to imitate the reality of the production and operation environment .

Which has achieved obvious effect in many aspects, such as fault location and investigation, project implementation plan designing and verification. And it also improves maintenance personnel technical level.

\section{Technical scheme}

Running a virtualized network platform to simulate additional equipment, changing the parameters of the equipment configuration, equipment downtime and other triggered the fault phenomenon, to restore the fault site. 
And the information capture tool is used to capture the related parameters. The contents include: equipment configuration information, interface information, information on the status of the device interface, status information of the shortest path first (OSPF), routing information of the shortest path first (OSPF), MPLS state information, LSP MPLS information, BGP state information, MPLS VPN BGP status information, VPN information, such as routing information.

On the virtual network platform, the interface and protocol parameters and state information of the corresponding device are obtained when the network fault occurs. Analysis of the causes of failure, fault location. Then come to the solution and verify it on the platform. The solution which passed the testing will Implement and verify in the real network.

\section{Case study}

A site equipment failure, resulting in a broken network and the impact of the station is near the regional station. For example, The Comprehensive data network of Chengde Power Supply Company includes Jianping station、station Pingquan、Xiaosigou station 、Daqing station 、 Liaohe station station and so on hand in hand forming a ring topology. Pingquan County station, Pingquan station, Xiaosigou station, Qi Gou station, Wang tufang station, Liaohe and Daqing station station have two links to the integrated data network of Chengde Power Supply Company. The Daqing station routing equipment downtime leads to Liaohe Station 、Wang Qi Gou station and Adobe station three station network interrupts. It needs about 3 minutes to the network automatic recovery. (As show in Figure 4: Fault range network topology)

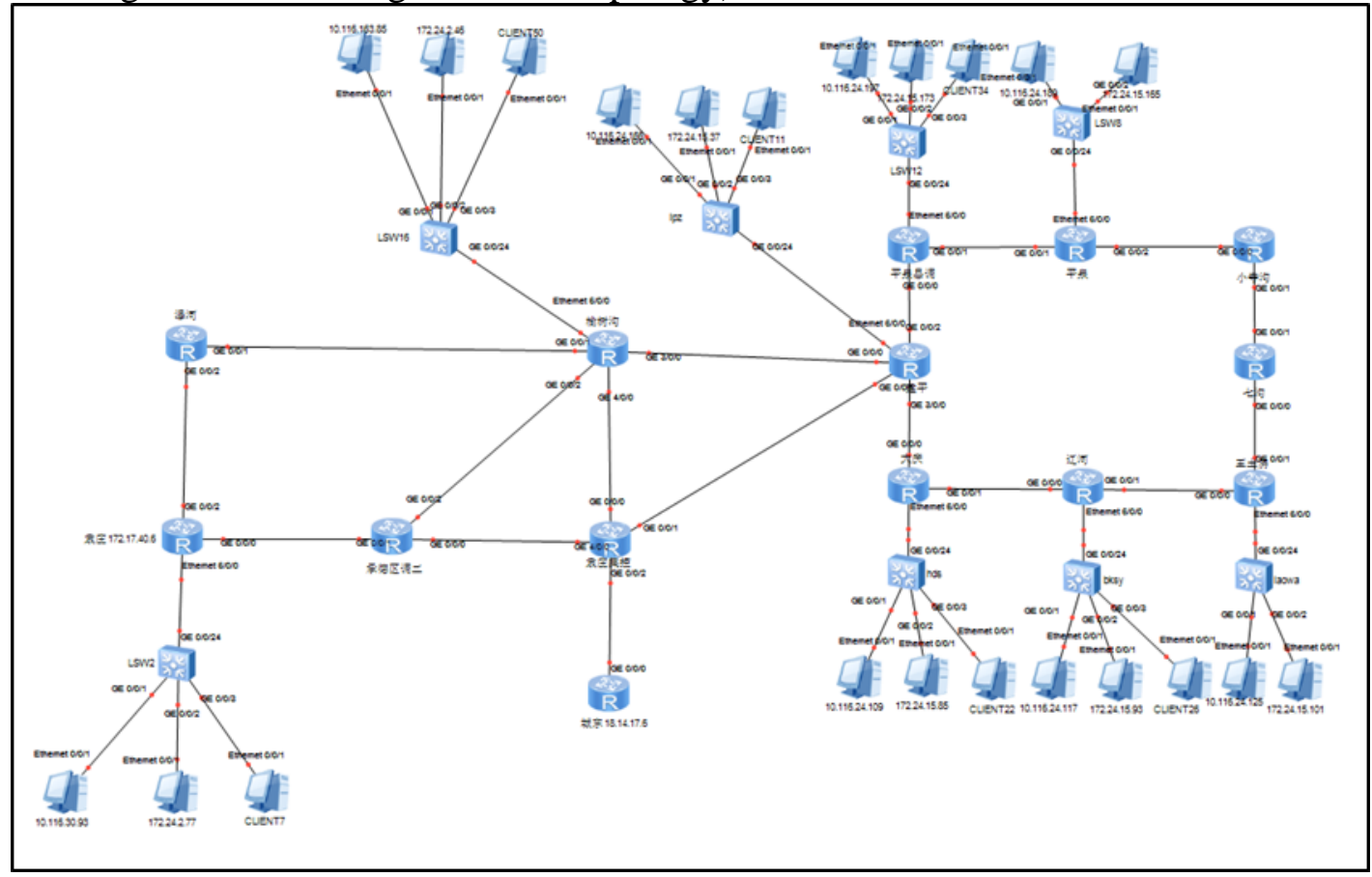

Figure 1: Fault range network topology 


\section{Case solution}

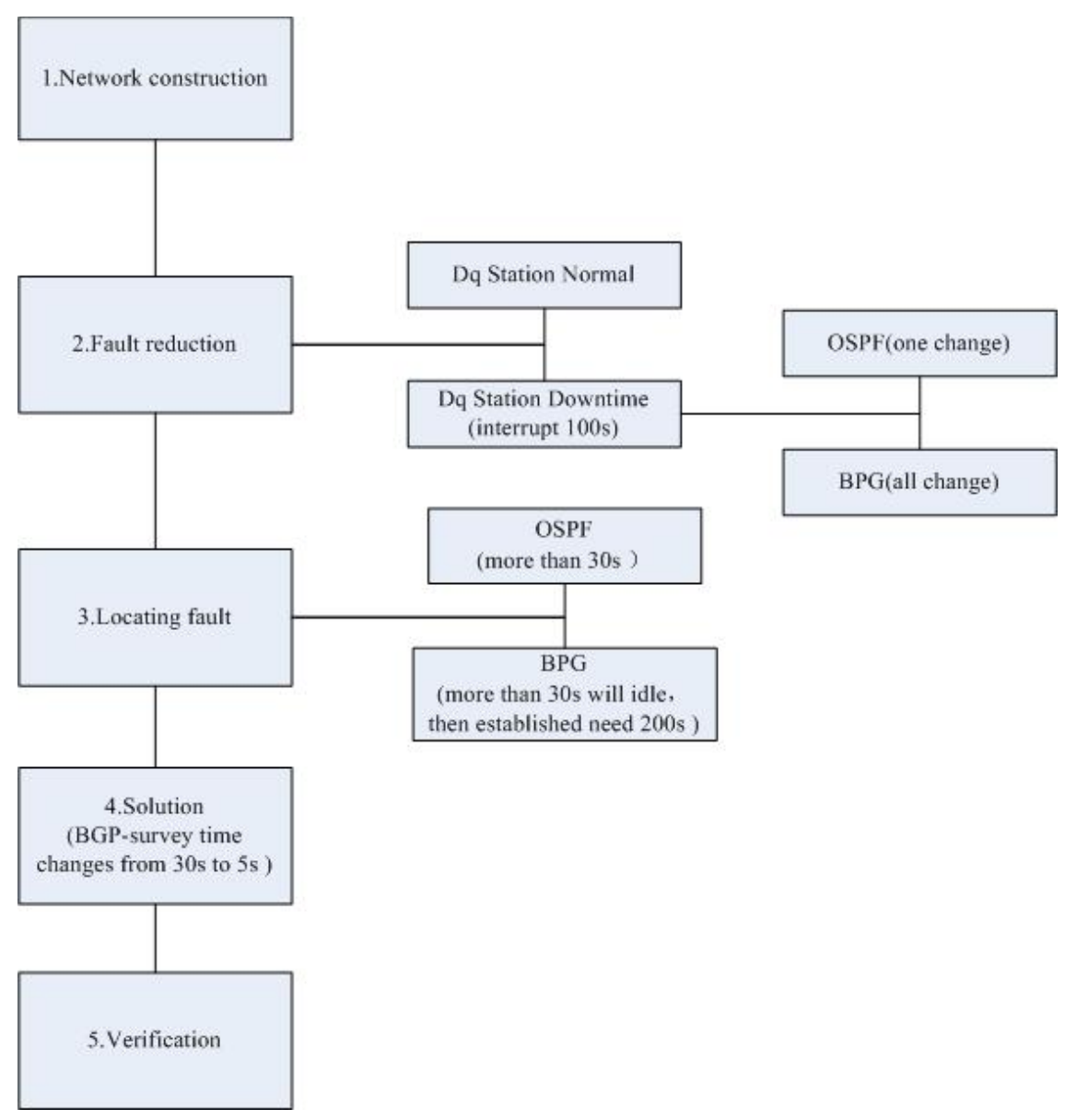

Figure 2: The process of the fault solution

The process of the fault solutionas show in the Figure 2:

\section{First ,network construction}

In the environment of the virtual platform, the equipment of the core layer andPingquan-county are built, reducing the company's real network environment.

\section{Second, Fault reduction}

The comparison and analysis of the stations near the three station equipment in the agreement of the operation and status information when the equipment normal work or downtime two situations (analog equipment downtime)of Daqing station.

When the network of Daqing station is normal, then detect the entries and directions of the route. The result is that the three paths are directed to the Daqing station.

When the Daqing Station equipment downtime, three stations interrupted about 100 seconds. Check the status and neighbor information of the three sites under different network protocols such as: Open Shortest Path First(OSPF) and Border Gateway Protocol(BGP). Results: neighbor state information of the three stations in the BGP changes, the status information of the border gateway protocol (BGP) is from the establishment to the idle and then by the idle state to the establishment state.

The neighbor state information of the three stations in the OSPF: only the Liaohe station state information changes in the OSPF. the neighbors of Liaohe River station is change from two OSPF neighbors which are Daqing and Wang Adobe to one OSPF neighbor the Wangtufang.

Check the timer parameters of Open Shortest Path First (OSPF) and the Boundary network protocol (BGP), the results are: Hello 10S, Death 40S,Polling 120S

Heavy 5S,Transmission delay $1 \mathrm{~S}$.

\section{Third,locating fault}

From the timer parameter in the second step of the BGP neighbor status is maintained for 
30s. The BGP neighbor information exchange through the OSPF routing, routing convergence time exceeds 30 seconds of the OSPF,the BGP neighbor state is changed from established to idle. After theOSPF routing convergence, BGP is to establish the neighbor relationship second time. Then the BGP routing is completed. The BGP routing to build a neighbor requires about 30S, coupled with the border gateway protocol (BGP) routing convergence time. The whole process takes about 200 seconds.Due to the above reasons analysis, Daqing station routing equipment downtime, three station network also interrupt and then restore automatically after 200 seconds (about 3 minutes).

\section{Fourth, solution}

The border gateway protocol (BGP) to keep the survival time from 30 seconds to 5 seconds. Reduce the survival time of the border gateway protocol (BGP), improve the neighbor survival detection time, thereby reducing the network interrupt time.

\section{Fifth , verification}

In the virtual network platform, the survival time of the border gateway protocol (BGP) changes from 30 seconds to 5 seconds. Through the system running, verify the network interruption time is greatly shortened, the success of the verification.

The sixth step: the actual production environment adjustment

Modify the reality production network equipment parameters, according to the step 5 of the border gateway protocol (BGP) keep alive time, when Daqing station network equipment downtime, network fault time narrowed to about 100s.

\section{Summary}

Virtual network platform can effectively restore the scene of the accident, and provide effective data support for operation and maintenance personnel analysis and processing, so as to quickly locate the fault point, reduce the time of fault response, improve the accident handling capacity and efficiency.Virtual network platform provides a simulation, effective technical tools for management and technical personnel, not only changing of the original management concept of fixed mode, but also greatly improve the network management level, improve a high availability platform for the technical personnel.

\section{References}

[1] Shi Bingxin, Liu Wei, XueFenghua. Application of expert system in network fault management [J].Data communications. 2000 (03)

[2] Liu Peng. Network fault diagnosis and exclusion [J]. Heilongjiang meteorology. 2004 (04)

[3] Wu Tiejun. Experience in network failure to talk about [J].Printing technology. 2000 (10)

[4] Tian Zhaojun. Network fault diagnosis and exclusion of [J].Computer Era. 2002 (02)

[5] Xu Zhanwen, Liu Xin Li, Hong. Network fault general solutions [J].. Journal of Shenyang Medical College 2002 (03)

[6]QiuXiaoli. The treatment of common network failures [J].Southern China financial computer. 2004 (10) 